MIST

PUBLICATIONS

\title{
IGES 5.0 Recommended Practices Guide
}

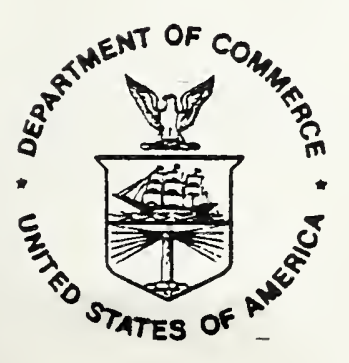

PC

100

rent of Commerce

.456 ture of Standards and Technology

no. 4600

g Engineering Laboratory

1991

c. 2 



\section{IGES 5.0 Recommended} Practices Guide

\section{IGES Recommended Practices Committee}

IGES/PDES Organization

Linda Martino, IBM Corp.

Chairman, IGES Recommended Practices Committee

Dennette A. Harrod, Jr., Computervision Corp.

IGES Project Manager, IGES/PDES Organization

William Conroy, NIST/EDS

Chaiman, IGES/PDES Organization

Joan Wellington

Editor, IGES/PDES Organization

May 1991

U.S. Department of Commerce

Robert A. Mosbacher, Secretary

National Institute of Standards and Technology

John W. Lyons, Director

Manufacturing Engineering Laboratory

Gaithersburg, MD 20899 


\section{ABSTRACT}

The IGES 5.0 Recommended Practices Guide is intended to assist both users and implementors of the Initial Graphics Exchange specification (IGES) Version 5.0. It contains two types of information: explanations of implementation practices that are alternatives to those documented in the specification, including preferred alternatives where preferences exist, and clarifications of ambiguities or omissions in the specification. Explanatory Recommended Practices address topics such as the explanation of complex areas in the specification, testing methods, processor performance considerations and the mapping of vendor entities into the set of IGES entities. Recommended Practices that clarify an ambiguity or omission are usually preludes to changes in the specification.

The Recommended Practices Committee of the IGES/PDES (Product Data Exchange using STEP) Organization (IPO) prepares the Recommended Practices Guide. Interested users and implementors from a variety of organizations prepare and donate material for the document. Suggestions for future topics or completed forms which propose new Recommended Practices (see Appendix A) should be sent to the Chairman of the Recommended Practices Committee in care of the IPO Chairman, National Institute of Standards and Technology, Bldg. 220, Rm. A127, Gaithersburg, MD, 20899.

The IPO Chairman's committee has reviewed each of these Recommended Practices for accuracy and acceptability to the general IGES community. In general, while preferred techniques are sometimes offered, no individual techniques are endorsed. The final choice of technique can be made only in conjunction with the details of the environment such as the system, the processor, and the application. 
INTRODUCTION •. • • . . . . . . . . . . . . . . . . 1

SUBMISSION PROCEDURE . . . . . . . . . . . . . . . 1

APPROVAL PROCEDURE . . . . . . . . . . . . . . . . 3

RP 1: DELIMITER . . . . . . . . . . . . . . . . . . 4

RP 2: WITNESS LINE SUPPRESSION . . . . . . . . . . . 5

RP 3: TRANSFORMATIONS OF NESTED SUBFIGURES • . • • . • . . . 6

RP 4: TRANSFORMATION MATRIX PROCESSING . . . . . . . . . 7

RP 5: SYSTEM ID PARAMETER . . . . . . . . . . . . . . 8

RP 6: MAGNETIC TAPE FORMAT . . . . . . . . . . . . . 9

RP 7: MAXIMUM COORDINATE VALUE ............. . 10

RP 8: INDEPENDENT WITNESS LINES . . . . . . . . . . . . 11

RP 9: POSTPROCESSOR DIAGNOSTICS . . . . . . . . . . . 12

RP 10: TEXT FONT 0 . . . . . . . . . . . . . . . . 14

RP 11: USER INTERFACE . . . . . . . . . . . . . . 15

RP 12: FLAG NOTE RESTRICTIONS . . . . . . . . . . 17

RP 13: VIEWS FOR TRANSFORMATIONS . . . . . . . . . . 18

RP 14: TRANSLATOR DOCUMENTATION . . . . . . . . . . . 19

RP 15: ZERO RADIUS ARCS . . . . . . . . . . . . . 20

RP 16: TRANSLATION VECTOR . . . . . . . . . . . . 22

RP 17: MODEL SPACE SCALE ................ . 24

RP 18: PROCESSING MACRO ENTITIES . . . . . . . . . . 26

RP 19: INDEPENDENT AND DEPENDENT PROCESSING . . • . . . . . 27

RP 20: BACK POINTERS IN VIEW ASSOCIATIVITY . . . . . . . 29

RP 21: COMMENTS IN PARAMETER DATA RECORDS . . . . . . . 30

RP 22: BOUNDED PLANES . . . . . . . . . . . . . . . 31

IGES 5.0 Recommended Practices Guide May 1991 V 
RP 23: RULED SURFACE DISCONTINUITY

RP 24: REPRESENTATION OF LINEAR STRINGS

RP 25: MINIMUM USER INTENDED RESOLUTION

RP 26: ARROWHEAD AND LEADER LINE DATA .

RP 27: IIMITATIONS ON LEVEL IDENTIFIERS

RP 28: SINGLE/DOUBLE PASS PROCESSING

RP 29: SPLINE CURVES AND SURFACES.

RP 30: GLOBAL PARAMETER DEFAULTS

RP 31: HIERARCHY FLAG

RP 32: ARROW AND WITNESS POINTERS

RP 33: ENTITY ORIGINS

RP 34: TEXT ROTATION ANGLE

RP 35: DUPLICATE TRANSFORMATION MATRICES

RP 36: EXCESS CHARACTERS IN REAL NUMBERS

RP 37: REDUCE I/O OVERHEAD

RP 38: UNIQUE SUBFIGURE NAMES

RP 39: EMPTY SUBFIGURES USED AS EXTERNAL REFERENCES

RP 40: SCALE IN MATRIX . . . . . . . . . . . . . 58

RP 41: ENTITY IDENTIFIERS . . . . . . . . . . . . 59

RP 42: UNIQUE NAMES . . . . . . . . . . . . . . 61

RP 43: CLOSED AREAS ................. . . 62

RP 44: TEXT BOX PROCESSING ............... . 63

RP 45: NULL TEXT STRING ................ 66

RP 46: DRAFTING SYMBOLS . . . . . . . . . . . . . 67

APPENDIX A. PROPOSED RECOMMENDED PRACTICES FORM . . . . . 69 
APPENDIX B. CHANGE ORDERS FOR IGES VERSION 5.0 • • • • • 71 Index . . . . . . . . . . . . . . . . . . . . . 80

IGES 5.0 Recommended Practices Guide May 1991 vii 
viii IGES 5.0 Recommended Practices Guide May 1991 


\section{INTRODUCTION}

The Initial Graphics Exchange Specification (IGES) Version 5.0 document specifies the structure and meaning of an IGES file. It is intended to be a concise explanation of the IGES file and entity formats and, in general, does not give detailed explanations of the underlying concepts or of the reasoning behind particular choices when alternative representations are available.

In addition, the specification document does not provide information on how to implement translators or on how to exchange data. This is especially a problem when decisions must be made for approximations. The Recommended Practices (RP) Guide is a collection of practices intended to help write translators. It is not a cookbook on how to write translators, but rather a collection of implementation issues that people have encountered. In most cases the practice will list a suggested solution. In some cases there is no suggested solution as more than one may be equally appropriate. It is still deemed helpful to alert implementors to each solution.

The Specification and RP Guide are meant to complement each other. Since RPs can be handled more quickly than Requests for Change (RFCS), some RFCs will be preceded by an RP. It is important to understand that RPs are NOT part of the Specification, but are guidelines; therefore compliance with them is NOT required.

Sometimes, an RP is used as the basis for an RFC, which ultimately generates an Edit Change order (ECO) to the Specification. When this happens, the corresponding RP becomes inoperative, and the next release of the RP guide reflects this fact; the RP number and Problem statement are retained, but the other sections, tables, and illustrations are deleted.

\section{SUBMISSION PROCEDURE}

This section explains how to prepare and submit a Proposed Recommended Practice (PRP) as well as the process a PRP follows while under consideration by the Recommended Practices Committee.

Anyone may submit a PRP. Submissions should be made on the form provided in Appendix $A$ of this Guide. Contents should conform to the following format. If approved, the PRP will be rewritten to ensure a uniform standard of clarity and grammar. 


\title{
Preparing the Form for a Proposed Recommended Practice
}

PRP Number: The PRP number and revision letter are assigned by the person responsible for RP change control. They help to keep track of the PRP as it progresses through the approval process.

Date: Use the date of the submission of the PRP. For a revision, use the date that the revision is submitted to the RP change control person.

Number of Pages: Include the number of pages to indicate the total length of the complete PRP.

Author's Name: Fill in the author's name and address so that questions and discussion can be directed to the appropriate person.

Affected processors: Show whether the PRP applies to preprocessors, postprocessors, both, or neither (write "none").

Category: Indicate which of the following standard categories for RPs the PRP addresses.

Software Practice
Conversion Method
Hierarchy Rule
Documentation
Performance
Testing Method

Software Practice Conversion Method Hierarchy Rule Documentation Testing Method

\author{
Implementation Rationale \\ Entity Mapping \\ Text \\ Workaround \\ IGES Description \\ Clarification
}

Affected Entities: List any IGES entities affected by the PRP. Use both the name and the entity number.

Reywords: List the major headings under which the PRP should be indexed. The RP manual includes an index of RPs by category and by keyword.

Topic: This item provides a title for the PRP. It should be concise without being cryptic.

Body of PRP: The rest of the PRP is the most important. It should be written to maximize the reader's understanding of the problem and of the proposed solution. The body should contain the following headings:

\author{
Problem statement \\ Solution \\ Rationale \\ A.ternatives Considered \\ Selected Methodology
}


Problem statement: The Problem Statement section should contain a clear, concise description of the problem that prompted the PRP. The description should be as general as possible, but should use examples where necessary to enhance clarity.

solution: The solution section should contain an abstract of the recommended solution to the problem. Give a detailed explanation with examples in the Rationale section.

Rationale: The Rationale section should contain an explanation of the alternative approaches considered, the recommended solution, and the reasons for selecting the recommended solution. Use examples liberally. The subheadings can be used to organize material in this section.

Alternatives considered: The Alternatives Considered section should contain a list of the different solutions developed. Examples aid clarity and should be used liberally. In addition to explaining the different alternatives, include a brief discussion of the advantages and disadvantages of each alternative, including the one eventually chosen.

selected Methodology: The Selected Methodology should contain the chosen alternative and an explanation of why it was selected. Justify the alternative here, and explain final details of the solution.

\section{APPROVAL PROCEDURE}

Completed forms which propose new Recommended Practices (see Appendix A) should be sent to the Chairman of the Recommended Practices Committee in care of the IPO Chairman, National Institute of Standards and Technology, Bldg. 220, Rm. A127, Gaithersburg, MD, 20899. The PRP will be distributed and discussed at the next meeting of the IGES Recommended Practices committee.

After discussion and possible modification, the Committee will either adopt or reject the PRP. If the PRP is adopted, it will be prepared for production and included in the next release of the Recommended Practices Guide.

The IGES RFC Review Committee, which is composed of the chairmen of the various IGES technical committees, will review the final version of each release of the Recommended Practices Guide and approve it for publication. 
Category: Software Practice

Reywords: Parameter Delimiter

Record Delimiter
IGES Document Version: All

Affected Processors: Both

Affected Entities: None

\section{Problem statement:}

Since it is likely that certain characters will be used to convey relevant information in the parameter data section, using these symbols for either delimiter character or end of parameter character will cause parsing difficulties in the postprocessor.

\{ This RP has been resolved by IGES Version 4.0 \} 
Category: Software Practice

Reywords: Blank Status

Annotation

Directory Entry
IGES Document Version: All

Affected Processors: Pre

Affected Entities:

Witness Line

(106, form 40)

\section{Problem statement:}

Witness lines used in annotation entities may be suppressed by two methods.

\section{Alternatives Considered:}

Do one of the following to suppress witness lines:

1. Place $a$ in the witness line pointer field of the annotation entity.

2. Set the blank status in the directory entry of the copious data entity.

Method 1 is confusing because during postprocessing it is impossible to determine whether the annotation entity lacks witness lines entirely or has blanked witness lines; it also prevents the IGES file from communicating the parameterization of blanked witness lines.

\section{Selected Methodology:}

Method 2 should be used whenever a witness line exists in an annotation entity, whether or not it is blanked. Method 1 should be used only if the witness line is not present in the annotation entity. 
Category: Hierarchy Rule

Reywords: Matrix Processing

Directory Entry

Subfigure
IGES Document Version: All

Affected Processors: Both

Affected Entities:

Subfigure Def. (308)

Subfigure Inst. (408)

Problem statement:

Before placement of a subfigure Instance during IGES postprocessing, any defining matrix and applied scale factors must operate on each entity. Where nesting (subfigure within subfigure) is used, the order of operations for scaling and translating must be consistent between preprocessors and postprocessors. The explanation in the specification is not sufficiently clear.

\{ This RP has been resolved by IGES Version 4.0 \} 
Category: Performance

Reywords: Identity Matrix

Directory Entry

Matrix Processing
IGES Document Version: All

Affected Processors: Pre

Affected Entities:

Transform. Matrix (124)

Problem statement:

The identity matrix in field 7 of a DE record can be identified in several ways.

Alternatives considered:

1. Use a pointer to an identity matrix (124).

2. Use the "default" value of 0 .

Selected Methodology:

For processing efficiency, it is preferable to use a zero in field 7 of the DE rather than to use a pointer to an identity matrix. 
RP 5: SYSTEM ID PARAMETER

Category: IGES Description

Reywords: Global Parameter 5

System ID

Global section
IGES Document Version: All

Affected Processors: Pre

Affected Entities: None

Problem statement:

Questions have arisen concerning the contents of Global Parameter 5, the System ID.

\{ This RP has been resolved by IGES Version 4.0 \} 
Category: Software Practice

Reywords: Tape Parity

ASCII Code

Tape Format
IGES Document Version: All

Affected Processors: Both

Affected Entities: None

\section{Problem statement:}

There is no standard for the exchange of IGES data on physical media such as magnetic tape.

\section{Alternatives Considered:}

Media to be used for data transfer should be agreed upon by the sender and receiver of the data. Possible methods include magnetic tape, floppy diskettes, or telecommunications.

\section{Selected Methodology:}

Based on ANSI X3.39-1973 and ANSI X3.22-1973, use magnetic tape with the following characteristics:

- One-half inch tape

Unlabeled

- 1600 BPI density

- 9 track

- 7 bit ASCII code (8th bit zero)

- 80 character fixed length records

- 10 records per tape block (This implies a block size of $800 ; 80$ character length * 10 records)

- A single end of file mark separates IGES data sets on tape

- Multiple consecutive end of file marks signal the end of tape data. 
Category: Software Practice

Reywords: Global Parameter 20

Max. Coordinate Value Global Section
IGES Document Version: All

Affected Processors: Pre

Affected Entities: None

\section{Problem statement:}

The preprocessor sets Global Parameter 20 to the largest value of the system, not to the largest value of the model.

\section{Selected Methodology:}

If possible, evaluate the model and determine the actual Maximum Coordinate Magnitude (MCM) in global space. Global Parameter 20 should be set such that:

$$
\begin{aligned}
& \text { any }|\mathrm{X}, \mathrm{Y}, \mathrm{Z}|<\mathrm{G} 20 \text { and } \\
& 0 \quad|\mathrm{G} 20|<1.2 \text { * MCM. }
\end{aligned}
$$

If the MCM is not calculated, do not set Global Parameter 20 to the maximum size of any model of the system; instead, use default parameter ("," "). 
Category: Entity Mapping

Reywords: Subord. Entity Switch

DE Level Number
IGES Document Version: All

Affected Processors: Both

Affected Entities:

Witness Iine

(106, form 40)

\section{Problem statement:}

Several systems have native database data structures in which the dimension witness lines are not linked to a "parent" entity (e.g., linear dimension, angular dimension). The IGES data files produced from these systems contain copious data (form 40) witness lines which are "independent" and dimension entities with the witness line pointers equal to zero. Conversely, many other systems have native database structures in which the "parent" entity always contains the references to witness lines and independent witness lines are not allowed. In moving an IGES file between these systems, especially from the former to the latter, an alternative mapping is needed to retain the witness line.

\section{Selected Methodology:}

Preprocessors should retain the "parent" structure whenever possible. Postprocessors for receiving systems which cannot support "independent" witness lines should map the copious data (106, form 40) to corresponding geometry entities (lines and arc segments). These entities should be placed on a graphics level (or associated with special attributes) to distinguish them from the legitimate model geometry.

An unused graphics level could be assigned by the postprocessor, or the graphics level could be selected by the user when the postprocessor is invoked. In either case, a message should be provided indicating the attributes which distinguish "witness line geometry" from "model geometry." If independent lines are used, the entity use flag should be set to "annotation." 
Category: Documentation

Reywords: Error Message

Postprocessor Robustness
IGES Document Version: All

Affected Processors: Post

Affected Entities: None

\section{Problem statement:}

IGES postprocessors often discontinue processing after an error is encountered in the file, and do not provide meaningful error messages.

\section{selected Methodology:}

The functions of an IGES postprocessor are similar to those of a compiler. The postprocessor should recognize errors encountered when an IGES file is processed, should provide the user with an indication of the error, and should, if possible, continue processing the file.

The key elements of a diagnostic capability are:

1. Determine seriousness of Error. The postprocessor determines the impact the error will have on the processing of the file, and categorizes the error. possible categories include FATAL ERROR, ERROR, WARNING.

2. Continue processing when Possible. After an error is identified, the postprocessor attempts to continue processing the file. Many errors will not prevent processing, and multiple errors that may exist will need to be identified. Options can be included to stop processing if a fatal error occurs, or if a specific number of errors occur, to avoid wasting CPU time when a file is not processable.

3. Provide Meaningful Error Messages. The postprocessor provides complete error messages that include a description of the error and the location of the error in the file. The postprocessor also should provide a summary description of the error and the actions taken, for display on a terminal.

The following $i$ a list of some common errors. This list is not com ete but contains a variety of errors. After each error message, the list contains a suggested 
action. The processor may need to take some corrective action before continuing after non-fatal errors. Always output some type of message to the user.

\section{POSSIBLE ERROR}

Global delimiters in error

Tape format--not ASCII

Binary section missing--binary format

Entity not supported

Global version not supported

No end of record delimiter

Terminate section missing

DE section missing

Sequence field

Out of order

Missing

Wrong format

Parameters

Wrong type

Invalid value

106--(IP parameter not

equal to $1,2,3$ )

Endpoints not on circle/conic

Pointers not in $D E$ range

out of range

\section{SUGGESTED ACTION}

Terminate

Terminate

Terminate

Bypass \& continue

Bypass \& continue

Bypass \& continue Continue

Terminate

Continue

Continue

Continue

Use default \& continue

Adjust \& continue

Adjust \& continue

Continue

Continue 
Category: Text

Reywords: Text Font

General Note
IGES Document Version: All

Affected Processor: Both

Affected Entities:

General Note (212)

Problem Statement:

Section 4.2 .9 of Version 2.0 of IGES states that "font 0 is an old symbol font and should no longer be used." At the same time, one of the philosophies of IGES development has been to maintain upward compatibility. These two items appear to conflict.

\section{Alternatives Considered:}

1. Eliminate the use of font code 0 by all preprocessors and postprocessors.

2. Support the acceptance of font code 0 by postprocessors only.

\section{Advantages/Disadvantages:}

Dis. \#1 Font code 0 was supported in version 1 of the document. Files may have been generated that already contain font code 0 . These files would no longer be processable.

Adv. \#2 Font code 0 would no longer be generated, but those files that already contain font code 0 would still be processable. Further, font code 0 could eventually be removed by processing the data back to IGES.

\section{Selected Methodology:}

Alternative 2 . 
Category: Documentation

Reywords: Summary Report

Information Message
IGES Document Version: All

Affected Processors: Both

Affected Entities: None

\section{Problem statement:}

Users are having difficulty determining the status of translators as they process and have little or no information on the actions completed by a processor.

\section{Alternatives Considered:}

The basic approach is to:

1. Provide a mechanism to inform the user that processing is continuing in a "normal" fashion.

2. Provide a summary report containing the entity types and counts processed at completion.

Item 1 can be addressed in an interactive session by sending a message to the workstation after each " $n$ " entities where " $n$ " could be 1 to 10. The message could include the DE pointer to verify that a loop situation has not occurred. Some implementations currently provide no clue as to how much (or how little) conversion has been completed.

Item 2 can be addressed by generating a summary report consisting of:

- translator name and version

- entities input, by type and count

- entities output, by type and count

- errors encountered, identified by entity type, count, condition, and DE pointer

- processing time (wall and CPU) 


\section{Selected Methodology:}

The items listed above suggest alternatives to solve this problem. The decision to include all items, a subset, or other indicators is left to the discretion of the implementor. 
RP 12: FIAG NOTE RESTRICTIONS

Category: IGES Description

Reywords: Flag Note
IGES Document Version: All

Affected Processors: Pre

Affected Entities:

Flag Note (208)

\section{Problem statement:}

A flag note with multi-line text causes problems in determining the size of the flag.

\{ This RP has been resolved by IGES Version 4.0 \} 
Category: Software Practice

Reywords: Extra View

Coordinate system

Matrix Processing
IGES Document Version: All

Affected Processors: Post

Affected Entities:

Transform. Matrix (124)

Problem statement:

The PDDI Report* found that several postprocessors create a separate view for each transformation matrix resulting in a proliferation of views.

\section{selected Methodology:}

The postprocessor should move the data to its appropriate position without creating a new view. This is even more important for conics since the transformation matrix is always required for moving conics to their final placement.

* Product Definition Data Interface, Task I - Evaluation \& Verification of ANSI Y14.26M Standard, IGES Committee Presentation by Booz, Allen \& Hamilton Inc. October 18, 1983. 
RP 14: TRANSLATOR DOCUMENTATION

Category: Documentation

Reywords: Manual

Error Message

Information Message
IGES Document Version: All

Affected Processors: Both

Affected Entities: None

\section{Problem statement:}

The PDDI Report identified documentation as a problem.

\section{Selected Methodology:}

The following is the minimum set of documentation:

1. Formal manuals:

- Installation and execution instructions

- Translation tables including entity support, mappings, approximations, and support limitations

- User options

- Error messages and any compensatory action.

2. Translation Messages:

- Error messages

- Informative messages

\section{EXAMPLES :}

A message indicating that a particular entity was not supported.

A message indicating that a font was not supported and another was substituted. 
Category: Software Practice

Reywords: Invalid Data

Circular Arc
IGES Document Version: All

Affected Processors: Both

Affected Entities:

Circular Arc (100)

\section{Problem statement:}

Some preprocessors write arcs of zero radius. This can occur for two reasons: (1) Either the user put a zero radius arc in his part, or (2) the magnitude of the coordinates of the center point was much greater than the radius of the arc, and precision errors caused the center point and endpoints of the arc to be coincident. of these two possibilities, it appears that precision problems are not nearly as common as user-generated zero radius arcs.

\section{Alternatives Considered:}

1. If the radius and center point of an arc are such that the radius cannot be properly represented with adequate precision, then the preprocessor should define the arc with its center at the origin, and use a transformation matrix to move the arc into its correct position.

2. If the user puts a zero radius arc in his part, it is invalid.

3. If a zero radius arc is encountered in the sending system's data base, the preprocessor should ignore it.

Alternatives 1 and 2 combined, allow the maximum amount of information to be transferred via the specification, and allow any presently existing IGES file to be read. Alternative 3 has the advantage that the entire burden is placed on the preprocessor; however it does not allow for the transfer of zero radius arcs. Existing files which contain zero radius arcs would not necessarily be readable under this proposal.

Although it is likely that zero radius arcs represent user or system error, it might be unwise to completely disallow their existence in IGES files. 
Selected Methodology:

Preprocessor:

Zero radius arcs are invalid in the IGES specification (see section 4.3, Version 5.0) and should not be written to the IGES file as an arc entity. An appropriate message should be issued.

Postprocessor:

If a zero radius arc is encountered, it should be skipped and an appropriate message should be issued. 
Category: Software Practice

Reywords: Transformation Matrix

Matrix Processing
IGES Document Version: All

Affected Processors: Post

Affected Entities:

Transform. Matrix (124)

\section{Problem statement:}

Postprocessor: Some systems do not handle translation vectors. Those systems that store a rotation matrix as part of the internal entity representation incur an additional processing burden in handiing translation vectors.

\section{Alternatives Considered:}

Use of the translation vector in preprocessing into the Specification is not to be discouraged. It is reasonable to assume that any preprocessor making use of this feature does so with justification. Correct postprocessing of IGES data involving a translation vector is to be encouraged. For receiving systems that store only a rotation matrix, correct postprocessing involves a computational overhead of a matrix multiplication and a vector addition. The rotation matrix in the IGES data remains as the correct rotation matrix in the receiving system. This is not considered a burdensome situation relative to the alternative position of recommending that translation vectors not be inserted in the IGES data.

\section{Selected Methodology:}

A procedure for postprocessing IGES data containing a translation vector is suggested.

\section{Rationale:}

Entity data expressed in the specification which utilizes both a rotation matrix and a translation vector is of the form

\section{$R \mathbf{x}+v$}

where $\mathbf{R}$ is the 3 by 3 rotation matrix, $\mathbf{v}$ is the translation vector, and $\mathbf{x}$ is the vector of definition space coordinates. For systems accommodating only a rotation matrix, the origin of 
definition space necessarily coincides with the origin of model space. If such a system is to postprocess entity data of the above type, for which the origins do not coincide, the definition space coordinates in the receiving system must be adjusted to absorb the translation vector.

To absorb the translation vector, the quantity $\mathbf{R x}+\mathbf{v}$ must be expressed as $R(x+u)$ for some vector $u$. Then, in the receiving system, $R$ is still the rotation matrix, and $(x+u)$ is the vector of definition space coordinates.

The vector $u$ is $R^{-1} v$. This can be seen from the following:

$$
R x+v=R x+R R^{-1} v=R\left(x+R^{-1} v\right)=R(x+u) .
$$


Category: Software Practice

Reywords: Global Parameter 13

Model space scale

Global Section
IGES Document Version: All

Affected Processors: Both

Affected Entities: None

\section{Problem statement:}

Clarification is needed on the interpretation of Model space Scale (PDDI Report, pg. 93).

\section{Selected Methodology:}

Preprocessor:

1. If the model in the CAD system is a scaled model (e.g., quarter scale), the preprocessor should assign the following values for Global Parameter i3:

$$
\text { Model Space Scale }=\frac{\text { Model Coordinate }}{\text { Real World Coordinate }}
$$

For example, for a one-quarter scale model, this model space scale is 0.25 . Coordinate values assigned by the preprocessor will be "model" coordinates.

2. If the model is not a scaled model, the preprocessor should assign Global Parameter 13 as 1.0. Coordinate values assigned by the preprocessor are "real world" coordinates.

Postprocessor:

1. If the postprocessor encounters a Global Parameter 13 value of 1.0 , coordinate values are in real world coordinates.

2. If the postprocessor encounters a Global Parameter 13 that is not equal to 1.0 , the postprocessor should provide the user with two options and inform the user of the option taken. 
Option 1: Create a scaled model: Coordinate values in the IGES file are model coordinates and should be used directly.

option 2: Create a full scale model: Coordinate values in the IGES files should be divided by Global Parameter 13 .

$$
\text { Native coordinate }=\frac{\text { IGES File coordinate }}{\text { Global Parameter } 13}
$$

Note: This Recommended Practice applies only to coordinate values. It does not apply to other values such as arrow height or text box height. 
Category: IGES Description

Reywords: MACRO PD Parameter

Parameter Data
IGES Document Version: All

Affected Processors: Both

Affected Entities:

MACRO Definition (306)
MACRO Instance
(600-699\& 1000-9999)

Problem Statement:

The syntax of the macros is considerably different from anything else in the specification. Specifically, the word MACRO in the parameter section caused some processing problems (PDDI Report, pg. 84 ).

\{ This RP has been deleted - its content contradicted IGES \} 
Category: Hierarchy Rule

Reywords: Subord. Entity Switch Directory Entry
IGES Document Version: All

Affected Processors: Both

Affected Entities: None

\section{Problem statement:}

There is confusion as to the meaning and use of the subordinate Entity Switch.

\section{Selected Methodology:}

Physical dependency means that the subordinate entity cannot exist in the native system unless the superordinate entity exists. Logical dependency means that the subordinate entity can exist alone, but that it will also be part of some sort of logical grouping in the native database. The implications of the above definition are as follows:

1. The matrix pointed to by the logically superordinate entity has no effect on the subordinate entity's physical location.

2. An entity cannot be physically and logically dependent upon the same superordinate entity.

3. When an independent entity is encountered, it should be immediately added to the native database.

4. When a physically dependent entity is encountered, it should not be added to the database, since it cannot exist alone. It will be processed and added to the database when its physically superordinate entity is processed.

5. When a logically dependent entity is encountered, it should be immediately added to the database since it can stand alone, but some mechanism should be devised so that when the logically superordinate entity is processed, the logically subordinate entity can be located in the native database and have the logical connection established. 
Below are several examples.

Subordinate Entity superordinate Entity

witness line conic

line

leader line

$\operatorname{arc}$ linear dimension subfigure definition group (402, forms 1, 7, $14 \& 15)$

radial dimension and group

subfigure definition and group
Relationship

physical

physical

logical

physical

logical

physical

logical 
Category: IGES Description

Reywords: View Associativity
IGES Document Version: All

Affected Processors: Both

Affected Entities:

Views Visible Assoc. (402, forms $3 \& 4)$

Problem statement:

The Views Visible Associativities (402, forms 3 \& 4 ) can be unnecessarily complex for a preprocessor to build. The term "related entities" means that the entities are to be associated with all the views pointed to by the view Associativity. The View Associativity points to the related entities in class 2 and the related entities point to the views associativity in the DE view pointer field.

\{ This RP has been resolved by IGES Version 5.0 \} 
Category: Software Practice

Keywords: Comment
IGES Document Version: All

Affected Processors: Pre

Affected Entities: All

\section{Problem statement:}

Comments may follow the end of record delimiter in the Parameter Data (PD) section. Implementors are reminded of this fact. A postprocessor should be able to bypass any comment records after the last PD parameter of the entity.

\section{Selected Methodology:}

The comment field starts after the end of record delimiter and may extend for several records. The Directory Entry (DE) back pointer field, section code ("P"), and sequence number are required on all PD records including comment records. The DE field 14 (PD line count) must include all comment records in the PD section. 
Category: Entity Mapping

Reywords: Bounded Plane

Bounding Curve

Unbounded Plane
IGES Document Version: All

Affected Processors: Post

Affected Entities:

Plane (108)

\section{Problem Statement:}

Some systems that do not support bounded planes nevertheless postprocess them as unbounded planes. In such cases there is a danger that the bounding curve, which is flagged as a subordinate entity, may not be postprocessed and that this information will be lost.

\{ This RP has been resolved by IGES Version 4.0 \} 
Category: Software Practice

Reywords: Composite Curve

Slope Discontinuous
IGES Document Version: All

Affected Processors: Both

Affected Entities:

Composite Curve (102)

Ruled Surface (118)

\section{Problem statement:}

A ruled surface that has a slope discontinuous composite curve as one of its defining curves will have a corresponding slope discontinuity.

\section{Alternatives Considered:}

1. Disallow use of a composite curve in defining a ruled surface.

2. Allow use of a composite curve in defining a ruled surface and issue an appropriate warning.

Selected Methodology:

Alternative 2 
RP 24: REPRESENTATION OF IINEAR STRINGS

Category: Entity Mapping

Reywords: Iinear spline Circular Arc
IGES Document Version: AII

Affected Processors: Both

Affected Entities:

\author{
Circular Arc (100) \\ Composite Curve (102) \\ Copious Data (106) \\ Line (110) \\ Parametric spline (112) \\ Parametric surface (114) \\ B-spline Curve (126) \\ B-spline Surface (128)
}

\section{Problem statement:}

In many systems, there are entities represented by Iines and "spars" displayed between a series of coordinates. These entities have no one-to-one counterpart in terms of naming conventions in the IGES specification. A common definition is proposed so that different vendors can successfully transfer this type of data between systems.

\section{Alternatives Considered:}

1. Use the Copious Data Entity (106) for entities consisting entirely of line segments;

Advantage: Simple to define, concise.

Disadvantage: Risks encouraging use as a catch-all for anything dealing with line graphics.

2. Use the composite Curve Entity (102) for entities which point to lines and circular arcs representing each segment. 
Advantage: Handles mixtures of lines and arcs.

Disadvantage: Complicated structure.

3. Use the Rational B-spline Entity.

Advantage: The definition of the IGES B-spline Entity includes a "class" field to describe it. This is the closest one-to-one entity correspondence not in the catch-all category.

Disadvantage: Creates longer entity definitions than the first two alternatives.

\section{Selected Methodology:}

Since the primary disadvantage of alternative 3 is longer processing time due to definition length, any reasonable alternative that takes considerably less processing time should be selected. Alternative 1 provides a clear, concise definition in a fraction of the space and executes in a fraction of the time taken by other options.

For entities consisting entirely of line segments, use alternative 1. For entities containing circular arcs or other curves, use alternative 2 . 
RP 25: MINIMUM OSER INTENDED RESOLUTION

Category: Performance

Reywords: Global Parameter 19

Minimum Resolution Global section
IGES Document Version: All

Affected Processors: Both

Affected Entities: None

\section{Problem statement:}

There is confusion as to the proper setting and use of Global Parameter 19 (Minimum Intended Resolution).

\section{Alternatives Considered:}

The description of the Minimum User-intended Resolution (MUR) in the IGES Specification, Version 5.0, states:

This parameter indicates the smallest distance in model space units that the system should consider as discernible. Coordinate locations in the file which are less than this distance apart should be considered to be coincident.

Coincident point testing is needed for identifying:

- a circle from a small arc

- a valid piecewise continuous composite curve

- a valid unit vector

- a valid line length

- valid start and terminate points lying on a conic or an arc.

- positional continuity requirements implied at the interior breakpoints of a parametric spline curve Entity (112). A breakpoint $\mathrm{T}(\mathrm{i})$ is on an interior breakpoint if $1<\mathrm{i}<n+1$ where the breakpoints of the parametric spline curve are $T(i),(i=1, \ldots, n+1)$ where the parameter $H$ has a value of 0,1 or 2 . Note the coincident point tolerance is not used to test for the slope continuity or curvature continuity. 
When testing for coincident points, two testing schemes could be used:

1. box metric, and

2. Euclidean metric.

The box metric testing scheme considers whether a point lies within a box (2-D square or 3-D cube) centered on another point. The sides are twice the value of the MUR. This scheme can produce inconsistent results depending on the orientation of geometry. An example of this is a horizontal line and a diagonal line, both with a size just larger than the MUR. The end points of the horizontal line would not be considered coincident, but the end points of the diagonal line would be considered coincident.

The Euclidean metric testing scheme considers whether a point lies within a radius (2-D circle or 3-D sphere) centered on another point. This scheme produces consistent results independent of the geometry's orientation. The expense of this scheme is that it involves squaring each delta coordinate, which slows down processing time.

It should be noted that some IGES processor implementors have no control over the coincident point testing schemes used in their system's geometric modelers.

\section{Selected Methodology:}

One coincident point scheme should be used throughout the IGES processor and its geometric modeler. It is more important that the preprocessor and postprocessor use the same method than which method is used. When both systems do not use the same method, errors may occur.

Each processor should document its coincidence testing method so users will be aware of possible critical discrepancies. 
Category: Entity Mapping

Documentation

Reywords: Leader Line

Arrowhead
IGES Document Version: All

Affected Processors: Both

Affected Entities:

Leader (214)

Witness Line

(106, form 40)

\section{Problem statement:}

IGES preprocessors and postprocessors handle leader lines and arrowheads differently. (PDDI Report, Task 1, pg. 71)

\section{Selected Methodology:}

Preprocessors should use the form number for the Entity Type 214 which matches the native arrowhead within a family. If the arrowhead in the native system does not match any IGES arrowhead, the preprocessor should select the Type 214 form number which most closely matches. The user should be informed, either in the user documentation or by means of a warning message, of the action taken.

Postprocessors should select the native arrowhead that matches the IGES arrowhead identified by the Type 214 form number. If the system does not have an arrowhead that exactly matches the IGES arrowhead, it should select a native arrowhead that most closely matches the IGES arrowhead. The user should be informed, either in the user documentation or by means of a warning message, of the action taken.

$\begin{array}{ll}\text { Family } & \text { Form \# } \\ \text { triangular } & 1,2,3,11 \\ \text { circular } & 5,6,12 \\ \text { rectangular } & 7,8 \\ \text { line } & 9,10\end{array}$


Category: Entity Mapping

Reywords: DE Level Number
IGES Document Version: All

Affected Processors: Both

Affected Entities: All

\section{Problem statement:}

Levels allowed on each system vary from 1 to $\mathrm{xxx}$ depending upon the vendor. Consequently, levels in excess of the maximum need a consistent way of being processed.

\section{Alternatives Considered:}

A variety of mapping methods have been proposed or are in use. They are examined below.

1. Wraparound. Entities on layer $x$ are mapped to layer $y$ where $\mathrm{y}=\operatorname{MOD}((\mathrm{x}-\mathrm{k}),(\mathrm{Ns}+1-\mathrm{k}))+\mathrm{k}$ where $\mathrm{k}$ is the lowest level and $\mathrm{Ns}$ is the number of supported levels on the receiving system.

2. Fill empty levels. A search is performed for levels whose number is less than or equal to Ns and which have entities assigned; assume Ne entity levels are found. The first $\mathrm{Ne}$ levels on which entities are defined outof-range are mapped to the empty levels. Remaining outof-range levels are mapped by alternative 1.

3. Ose nearest level. Out-of-range levels are mapped to the highest and lowest supported levels, respectively.

4. User defined. The user explicitly specifies the mapping relationship between levels by creating or referring to a mapping table.

\section{Selected Methodology:}

It is suggested that the user be given the capability of choosing from options 1 to 4 , the default method being wraparound. The postprocessor should inform the user of the mapping relationship it has applied. 
Category: Software Practice

Reywords: Multiple Pass

Single Pass
IGES Document Version: All

Affected Processors: Post

Affected Entities: All

\section{Problem statement:}

Is it more appropriate to process an IGES file with a single or multiple pass approach? The concern is whether a single-pass postprocessor can completely process all of the data.

\section{Selected Methodology:}

To preserve functionality, a postprocessor cannot process structure in a single pass. Therefore, multiple passes are recommended. 
Category: Entity Mapping

Reywords: Spline Curve Spline Surface
IGES Document Version: All

Affected Processors: Pre

Affected Entities:

$$
\begin{aligned}
& \text { Parametric Spline (112) } \\
& \text { Parametric Surface (114) } \\
& \text { B-spline Curve (126) } \\
& \text { B-spline Surface (128) }
\end{aligned}
$$

\section{Problem Statement:}

Parametric Spline Curve and Surface Entities need to be enhanced so (1) they may reside in an arbitrary dimensional image space, and (2) their underlying polynomials may be of arbitrary degree.

\section{Alternatives considered:}

1. Extend and enhance the Parametric spline Curve and Surface Entities (112, 114).

2. Extend and enhance the Rational B-spline Curve and Surface Entities $(126,128)$, while providing algorithms to convert between Parametric spline and Rational Bspline Curve and Surface Entities of arbitrary dimensionality and degree.

\section{Selected Methodology:}

Use Rational B-spline Entities (126, 128) instead of Parametric Spline Entities (112, 114) unless considerations dictate otherwise.

\section{Rationale:}

1. Parametric Spline Curve and Surface Entities (112, 114) can be fully captured without approximation by the Rational B-spline Curve and Surface Entities (126, 128) which are more general.

2. To avoid redundancy, Ent :y Types 112 and 114 will be corrected but not. enhance. All enhancements will occur 
within the context of the Rational B-spline Entities $(126,128)$.

3. Coded procedures are available from the National Institute of standards and Technology. These procedures translate between arbitrary dimensional, arbitrary degree, rational spline curves and surfaces defined by Parametric Spline Entities (112, 114) and Rational Bspline Entities (126, 128).

These procedures enable those with implementations in their native databases of parametric spline-like entities to use IGES Rational B-spline Entities (126, 128).

4. Rational B-spline Entities already allow for polynomials of arbitrary degree and will be enhanced to allow for multi-dimensional image spaces. 
Category: Software Practice

Reywords: Global Parameter

Default Value

Required Parameter
IGES Document Version: All

Affected Processors: Both

Affected Entities: None

\section{Problem Statement:}

There is a great deal of confusion and inconsistency in assigning default values for parameters in the Global section of an IGES file. There are also diverse opinions on which of the parameters are required. This Recommended Practice will show which parameters are required, and the default value which may be assumed for each parameter that is not required.

\section{Selected Methodology:}

The table on the following page shows, for each parameter in the Global section, whether the parameter is required, and if not required, the default value to be assumed. This table should be used by preprocessors in determining which values to include, and whether the assumed default values are appropriate. The table should be used by postprocessors in determining which default values to assume in the absence of a parameter value. If a required parameter is not supplied, the postprocessor may have to abort its processing, depending on the critical nature of the data. 
1. Parameter Delimiter

no

2. Record Delimiter

3. Product ID from sender

no

4. File Name

yes

(1)

5. System ID

6. Translator Version

7. No. Bits for Integer

8. Single Precision Magnitude

9. Single Precision Significance

yes

yes

yes

yes

yes

10. Double Precision Magnitude

11. Double Precision Significance

no

", " "

none

none

none

none

none

none

none

none

none

12. Product ID for Receiver

13. Model Space Scale

14. Unit Flag

15. Unit Description

16. Max. Number of Line Weights

17. Size of Max. Line Width

18. Date/Time File Generated

19. Min. User Intended Resolution

no

GP3

yes

(3)

(5)

no

yes

1.0

20. Approx. Max. Coordinate Value

yes

no

none

21. Name of Author

no

(4)

1

0.0

none

none

none

none

no

none

23. IGES Version

no

3

24. Applicable Drafting Standard

25. Date/Time Model Created

no

0

no

none

(1) Required if External Reference used

(2) Required if double precision used

(3) Required if GP14 = 3

(4) String appropriate for GP14

(5) Required if line weight is significant

\section{Table Item Description:}

The information contained in the above table is the result of many hours of discussion. In the paragraphs which follow, each parameter will be defined and discussion will be presented of either the need for the parameter or the reasons behind the default value chosen.

1. Parameter Delimiter. This parameter defines the character to be used to separate parameters in a list in a free format record (i.e., in the Global and Parameter Data Sections). It is not required, and its default value is established by the IGES document as the string ("1H, "). 
2. Record Delimiter. This parameter defines the character to be used to signify the end of a parameter list in free format records ( $i . e .$, in the Global and Parameter Data sections). It is not required, and its default value is established by the IGES document as the semicolon ("1H;,").

3: Product ID from sender. This parameter contains the name of the model identifier (e.g., part name or number) on the sender's system which contains the model described in this IGES file.

4: File Name. This parameter contains the name of the datia file on the sending system which contains this IGES file. It is not always used by the receiving system, and is required only when the IGES file will be the object of an external reference.

5: system Identification. This parameter contains the vendor's name and model designation for the sending system. This information is required. The sender's system identification can be very useful in determining how to handle error conditions if they occur during the processing.

6: Translator Version. This parameter consists of the version number of the vendor's IGES preprocessor used to create this IGES file, and is required. Iike the system identification, it can be useful in determining how to handle processing errors.

7: Nuber of Bits Used to Represent Integer Values. This prameter is required.

8-11:

Floating Point Values Range. Parameters 8 and 10 are powers of 10 which represent the maximum value. Parameters 9 and 11 are the number of decimal digits of significance which can be actually represented on the sending system. Parameters 8 and 9 are always required and parameters 10 and 11 are required if double precision numbers are included in the file.

12: Product Identification for Receiver. This parameter can provide a receiver's product ID (e.g., part name or number). It is not required, and if not present, the contents of Parameter 3 (Product ID from sender) may be used.

13: Model space scale. This parameter defines the ratio between the size of items in the IGES file and the full 
size of the physical object. It is not required, and if not present, is assumed to be 1.0 .

14: Unit Flag. This parameter, along with Parameter 15, identifies the units used in creating the file. A value for this parameter is required.

15: Unit Description. This parameter gives a string equivalent of the units specified by parameter 14 . It is only required when parameter 14 has a value of 3 . If, in the other cases, no value is present, the postprocessor may assume the string which corresponds to the value of Parameter 14 .

16: Maximum Number of Line Weights. This defines how many different line weights are used in the IGES file. In many cases, there is no significance to line weights and, therefore, the value for the parameter is not required. If this parameter is not specified, a value of 1 is assumed.

17: Size of Maximum Line Width. This parameter defines the width of the widest line used in the IGES file. It is required only if the value for Parameter 16 is present. Further, a value of 0 here means to interpret the line weight parameter as a relative number. The relative line weights are useful on a system which strokes lines: a line weight of 3 indicates that 3 standard lines are to be drawn side by side to make the final line. This is not to be used in lieu of the line-widening property.

18: Date and Time of Exchange File Generation. This is the date and time that the preprocessor created the IGES file. It is required to help uniquely identify the IGES file both on the sending and receiving systems.

19: Minimum User Intended Resolution. This parameter defines the intended accuracy of the model. It is required. See RP 25.

20: Approximate Maximum coordinate value. This value indicates the size of the model to the postprocessor. This information is necessary on some systems which have to set a scaling factor for the model before the postprocessing can begin. If a reasonable value cannot be placed in this parameter by the preprocessor, it is better to indicate that a default value is to be used for the parameter (",",) than to use an arbitrarily large (but unreasonable) value. If the default indication is used, then the postprocessor is responsible for determining the necessary value. See RP 7 . 
21: Name of Author. This information is strictly for documentation purposes. Its value to the receiving system depends on the contractual arrangements made between the sending and receiving organizations. It is not required and no default value is defined.

22: Organization. This information is strictly for documentation purposes. Its value to the receiving system depends on the contractual arrangements made between the sending and receiving organizations. It is not required and no default value is defined.

23: IGES Version. This parameter tells the postprocessor which version of the IGES specification was used to generate the file. The information is useful to the postprocessor for determining whether or not it may run into data forms or IGES features which it cannot handle. For reasons of upward compatibility, this parameter is not required. If not present, the postprocessor may assume that the Version 2.0 specification (parameter value of 3 ) is indicated.

24: Applicable Drafting standard. This information indicates that the creator of the model in the IGES file complied with a specific drafting standard while creating that model. The postprocessor will probably not modify any of its processing based on this parameter, but the information may be useful to the receiving system in ensuring that drafting entities added to the postprocessed model will comply with the same standard. The parameter is not required, and its default value is 0 (no standard specified).

25. Date/Time Model Created/Modified. Time model created or modified, whichever occurred last. Preprocessors may use the default $(",, ")$ if unknown. (V.5.0) 
Category: Hierarchy Rule

Reywords: DE Hierarchy Flag

Directory Entry
IGES Document Version: All

Affected Processors: Pre

Affected Entities: All

Problem statement:

Use of the Hierarchy Flag on an entity-by-entity basis is unclear. The Hierarchy Flag set to 0 in the view, drawing, or group could cause a postprocessor to ignore the line font, level number, view pointer, blank status, line width and pen number in all entities within a view, drawing or group.

Selected Methodology:

( This RP has been resolved by IGES Version 5.0 ) 
RP 32: ARROW AND WITNESS POINTERS

Category: Software Practice

Reywords: Witness Line

Arrowhead
IGES Document Version: All

Affected Processors: Pre

Affected Entities:

$$
\begin{aligned}
& \text { Angular Dim. (202) } \\
& \text { Linear Dim. (216) }
\end{aligned}
$$

\section{Problem Statement:}

Dimension entiti often contain two (2) leader/arrow entity pointers and two 2) witness line entity pointers. They are usually referred 0 as ARW1, ARW2, WIT1, and WIT2. One would normally assume that ARW1 corresponds to WIT1, etc., and some postprocessors measure the distance from the head point of the arrow to the end point of the witness line to calculate an "overshoot" for their internal database representation.

There is no requirement in IGES that this assumed relationship be maintained by the preprocessor. This information can, in fact, be established by computational examination; however, this places a needless burden on the postprocessor.

\section{Selected Methodology:}

The preprocessor should support the assumed relationship when writing an IGES file. 
Category: Software Practice

Reywords: Transformation Matrix

Entity origin

Identity Matrix
IGES Document Version: All

Affected Processors: Pre

Affected Entities:

General Note (212)

Text Display (312)

Subfigure Inst. (408)

View (410)

\section{Problem statement:}

There is a problem in processing the General Note (212) and Singular subfigure Instance (408) Entities. These entities contain explicit "origin" fields in their Parameter Data. However, any entity can be repositioned by providing a translation vector in an associated Transformation Matrix (124).

Some preprocessors set the Parameter Data for the origin to $(0,0,0)$, and associate a Transformation Matrix Entity with the identity rotation matrix and a $(X, Y, Z)$ translation to locate the entity. Some "simplistic" postprocessors are not designed to look for an associated translation, and consequently misplace the entity.

Worse, some preprocessors place the $(X, Y, Z)$ in both the Parameter Data section and a Transformation Matrix; the corresponding postprocessor obviously ignores one of the values, but other postprocessors misplace the entity. Since both must be applied, the data should not be duplicated; this practice is, in fact, incorrect.

The problem also applies to the Text Display Template (312), and the View (410), which has an (X,Y) translation in the parent Drawing (404). In fact, this practice can be generalized to any current and future entities which contain an explicit origin in their Parameter Data section. 


\section{Selected Methodology:}

The preprocessor should place the entity's origin in the appropriate field of the Parameter Data section, and set the translation vector in any associated Transformation Matrix (124) to $(0,0,0)$.

However, there are legitimate reasons for valid data to be contained in both places, so all postprocessors must be prepared to handle combined situations. 
Category: Software Practice

Reywords: Transformation Matrix

Rotation Angles
IGES Document Version: All

Affected Processors: Pre

Affected Entities:

General Note (212)

Text Display (312)

\section{Problem statement:}

There is a problem in processing the General Note Entity (212). This entity contains explicit "rotation angle" and "mirror" fields in its Parameter Data. However, any entity can be reoriented by providing a rotation matrix in an associated Transformation Matrix (124).

Some preprocessors set the Parameter Data for the rotation to zero, and associate a Transformation Matrix Entity with the desired rotation matrix and a $(0,0,0)$ translation to locate the entity. Some "simplistic" postprocessors are not designed to look for an associated rotation, and consequently misplace the entity.

The problem also applies to the Text Display Template (312). In fact, this practice can be generalized to any current and future entities which contain an explicit rotation angle in their parameter data.

\section{Selected Methodology:}

The preprocessor should place the entity's rotation in the appropriate field of the Parameter Data section.

However, there are legitimate reasons for valid data to be contained in both places, so all postprocessors must be prepared to handle combined situations. 
Category: Software Practice

Reywords: Transformation Matrix

Entity origin

Rotation Angles
IGES Document Version: All

Affected Processors: Pre

Affected Entities:

Transform. Matrix (124)

\section{Problem statement:}

Some preprocessors associate a Transformation Matrix (124) with every Circular Arc (100) even when they contain identical data.

For example, holes on a flat surface have a common normal vector, but the preprocessor gives each circle its own (identical) 124 instead of pointing at a common one. Another problem is that the preprocessor may set the origin in the Parameter Data section to $(0,0)$ and generate $124 \mathrm{~s}$ with identical rotation matrices but unique translation vectors.

In a slightly different vein, some preprocessors create circular Arc entities such that the start angle is always zero ( $Y 2=Y 1$ ), and create a Transformation Matrix which rotates it into position. In the case of the filleted corners of a cut-out on a planar surface, they could all use the same Transformation Matrix if their start and end points are adjusted.

Either of these implementations leads to needlessly large IGES files, and places a processing burden on the postprocessors.

Although the circular arc is used to illustrate the problem it also occurs in matrices associated with Subfigure Instances (408), dimension entities (200 series), and other geometric entities.

\section{Selected Methodology:}

The preprocessor should keep track of the Transformation Matrices it has generated and point to them instead of creating new ones.

However, there are legitimate reasons for creating matrices with a common normal vector; Conic Arc Entities (104) on the same plane but with different rotation angles are one example. 
RP 36: EXCESS CHARACTERS IN REAL NUMBERS

Category: Software Practice

Reywords: Number Format
IGES Document Version: All

Affected Processors: Pre

Affected Entities: All

\section{Problem statement:}

Some preprocessors output real numbers in a fixed-length format which takes up a lot of space; in some cases, only two (2) real numbers can be placed on a line in the Parameter Data record.

\section{Selected Methodology:}

Preprocessors should use the following techniques to reduce the length of text-string representations of real numbers in the Parameter Data record.

1. Delete leading blanks.

2. Delete trailing zeroes.

3. Round coordinate values to Global Parameter 19 (Minimum User-Intended Resolution). Coefficients should not be rounded.

4. Avoid unnecessary use of scientific notation. 
Category: Software Practice

Reywords: Thrashing
IGES Document Version: All

Affected Processors: Post

Affected Entities: All

\section{Problem statement:}

A postprocessor can waste a lot of time thrashing the disk while trying to read an IGES file. The problem is caused by the Directory Entry records and Parameter Data records of an entity being physically distant from each other.

Consider the processing of a Linear Dimension Entity (216). The lines which contain the DE are read into a system $I / O$ buffer (from $2 \mathrm{~K}$ to $8 \mathrm{~K}$ in length). Reading the lines of the associated PD record will almost certainly over-write the I/O buffer. These lines contain pointers to DE lines that were probably already in the buffer that just got over-written. similarly, reading the next DE will over-write $P D$ records of the subordinate entities that were read into the buffer along with the parent's PD record. Worst case (all pointers non-zero) implies twelve (12) physical reads of the disk, and the postprocessor will probably get swapped-out while waiting for the I/O.

\section{Selected Methodology:}

The postprocessor could open the IGES file with two (2) logical unit numbers; one for reading $D E$ lines, and one for reading $P D$ lines. The system will assign each its own I/O buffer, thus avoiding the over-write problem. Since there will be fewer physical reads, the translator should stay in-core for longer periods of time, and the translation should proceed much faster. 
Category: Software Practice

Reywords: Subfigure
IGES Document Version: All

Affected Processors: Both

Affected Entities:

Subfigure Def. (308)

Network Subf. Def. (320)

\section{Problem statement:}

Three variables in the IGES definition of subfigures may cause problems when exchanging data. First, IGES does not specify a naming convention for subfigures (PD field 2 of entities 308 and 320). Second, IGES does not require that a subfigure definition only appear once in any IGES file. Third, every CAD/CAM system has its own unique naming conventions for subfigures, and it cannot be assumed that a receiving system can attach any significance to these names.

Combined, it is possible for a postprocessor that wants to use the name of the subfigure, but only recognizes the first six characters, to misinterpret. the information and either (a) process one definition and ignore another, or (b) process a definition and then overwrite it with a different definition.

\section{Alternatives considered:}

1. The preprocessor shall use a naming convention that makes each subfigure name unique within an IGES file. Upper- and lower-case characters shall not be mixed, and symbols represented by the ASCII values 0-37 octal shall not be used at all. The naming convention will provide uniqueness in the first six characters.

The postprocessors shall process every subfigure in the IGES file. If it requires a unique name for the subfigure, then it shall check that the name has not been used before. If the name is a duplicate, then the system may respond in one of three ways:

a. Ignore the definition and inform the user of the action. 
b. Change the name automatically and inform the user of the action.

c. Ask the user what to do.

2. The preprocessor can use any naming convention that it desires, but it shall only create one uniquely named IGES subfigure definition for each subfigure defined in the model.

The postprocessor shall always assume that every subfigure definition in the IGES file is unique to that file, and must be processed to capture all required information. The postprocessor shall provide a mechanism to make the subfigures just processed unique to the model created from the IGES file, such as mapping unrecognized characters to recognizable characters.

\section{Selected Methodology:}

Alternative 2 is the most accommodating approach. In both alternatives, the idea is to create subfigures that belong strictly to one IGES file at a time. Therefore, it is better not to restrict the preprocessor because there might still be conflicts between naming conventions on different systems. It also puts less of a burden on the postprocessor as it does not have to assume that the subfigure might already exist on the system and check for it. 
Category: Software Practice

Reywords: Subfigure

Required Pointers
IGES Document Version: All

Affected Processors: Both

Affected Entities:

Subfigure Def. (308)

\section{Problem statement:}

Some IGES preprocessors are creating "empty" Subfigure Definitions (308), i.e., Parameter Data field 3 (number of entities in definition) is zero. This should not be confused with subfigure definitions that consist exclusively of entities which are not supported by the postprocessor.

In some cases, the purpose is to exchange library subfigures, with the expectation that a subfigure definition with no pointers causes the postprocessor to check for geometry already in the system under the same name. This can cause problems for postprocessors not recognizing this mechanism. The postprocessor may abort, or subsequent attempts to manipulate the translated data may cause fatal errors.

This is an incomplete IGES file, and is an unacceptable alternative to the External File Reference mechanism for passing library subfigure data.

\section{Selected Methodology:}

Exchange library subfigures using the External File Reference Entities and not through empty subfigures. 
Category: Software Practice

Reywords: Transform. Matrix
IGES Document Version: All

Affected Processor: Pre

Affected Entities:

Transform. Matrix (124)

\section{Problem statement:}

Some preprocessors have tried to convey the scale of an entity by embedding it in the coefficients of the entity's Transformation Matrix (124). This has been seen in the case of the Subfigure Instance (408) and View (410), neither of which are "geometric" entities. This probably is a consequence of the system's internal data representation; the determinant of such a matrix is equal to the scale, and it saves the four (or eight) bytes of an extra floating point number.

\section{Selected Methodology:}

Scale factor cannot legally be placed in the Transformation Matrix (124).

Multiplying the coefficients of a Transformation Matrix by a scaling factor is a violation of the specification, which states that the determinant of a form 0 or form 1 matrix (associated with geometry entities) must be either positive one or negative one, and that the columns constitute unit vectors.

Scale factor for the Subfigure Instance (408) and View (410) must be placed in the explicit scale field. 
Category: Software Practice

Reywords: Names
IGES Document Version: All

Affected Processor: Both

Affected Entities: All

\section{Problem Statement:}

Passing of entity identifier information between systems is confusing. Entity identifiers also are known as entity tags, names, or labels. The concept of identifiers allows users to identify entities in the sending system and to track them in the receiving system. IGES has the following methods:

- Name in PD section (subfigure definition \& network subfigure definition)

- DE label \& subscript fields

- Name property

The name property was added to IGES because the DE fields were too restrictive in size. The subscript field can contain only numbers (length 8) and the label field can contain alphanumeric data (length 8 ). The name property is unrestricted but adds a great deal of size to the file because it is a separate entity. The character string is the only useful piece of information.

The other problem has to do with uniqueness. Originally, many CAD systems did not allow the users to tag entities so preprocessors created unique tags. These unique tags did not help users know what native element in the original system became what entity in the receiving system. It also is not reasonable to keep uniqueness if there is a one-to-many mapping when the purpose is to track entities through multiple translations. In any event, IGES does not require uniqueness; therefore, it should not be assumed.

\section{Alternatives Considered:}

1. For subfigures, use PD parameters; for all other entities, use the name property and not the DE fields. This alternative would specify only one method for any entity type, but would increase file size.

2. Fill in the fields in a specified order, using only one method for a particular entity. 
Preprocessor order:

a. For subfigures, use PD parameters.

b. For all other entities, if the label fits into the DE fields, use those.

c. If not, use the name property.

Postprocessor order: The order is different from the preprocessor, as both the name property and DE fields may exist. If they both exist, it is reasonable to assume the name property is the more accurate data.

a. For subfigures, use PD parameters.

b. Use the name property if it exists.

c. Use the DE fields.

\section{Selected Methodology:}

Use method 2. This is the most complex, but it is efficient in terms of file storage while still allowing complete flexibility in name length.

Postprocessors must also handle non-unique names as uniqueness is not required by IGES. 
Category: Software Practice

Reywords: Names, Subfigures
IGES Document Version: All

Affected Processor: Post

Affected Entities:

Subfigure Def. (308)

Network Subf. Def. (320)

Name Prop. (406, form 15)

\section{Problem statement:}

IGES files contain names such as the subfigure Definition (308) and the Name Property (406, form 15). Many operating systems and applications have restrictions on what constitutes a valid name for files and internal names, but IGES does not contain any restrictions. Examples of system dependent restrictions are:

Names restricted to seven characters;

Names cannot contain underscores or special characters;

Names must be unique.

\section{Selected Methodology:}

Postprocessors should be aware of restrictions and should implement a substitution or mapping algorithm to convert invalid names to valid names.

\section{Rationale:}

Preprocessors can't be restricted to all possible restrictions for postprocessors. Postprocessors must be robust enough to map all names. 
Category: Software Practice

Reywords: Closed Areas
IGES Document Version: All

Affected Processor: Pre

Affected Entities:

Copious Data (106)

Sectioned Area (230)

\section{Problem Statement:}

Preprocessors use Copious Data Entities (106, form 11) to create closed areas such as curves which are referenced by the sectioned Area Entity (230).

\section{Selected Methodology:}

Preprocessors should use Copious Data Entities (106, form 63) to create closed areas.

\section{Rationale:}

Entity Type 106, form 63 includes a function that is lost with the form 11 since form 63 says it is closed and form 11 does not. The Entity Type 230 must point to geometry which is closed. 
Category: Software Practice

Reywords: Text
IGES Document Version: All

Affected Processor: Post

Affected Entities:

General Note (212)

\section{Problem statement:}

Systems having fixed aspect ratios for their text have difficulties creating a text string that stays within the box height and width defined by the IGES 212 entity (see Figure 1). This problem can cause the text to overwrite surrounding geometry or text. (Systems with variable aspect ratio text do not have this problem and may disregard this recommended practice.)

\section{Alternatives Considered:}

Three methods can be used to create text on systems with fixed text aspect ratios:

1. Use both the box height and width from the IGES 212 entity and adjust text to maintain both height and width (see Figure 1).

2. Use the defined box width from the General Note Entity (212) for the text width. This usually will result in text looking like Figure 3, but can sometimes result in the string exceeding the IGES box height.

3. Use the defined box height from the IGES 212 entity for the text height. This usually will result in the string exceeding the IGES box width (see Figure 2). 

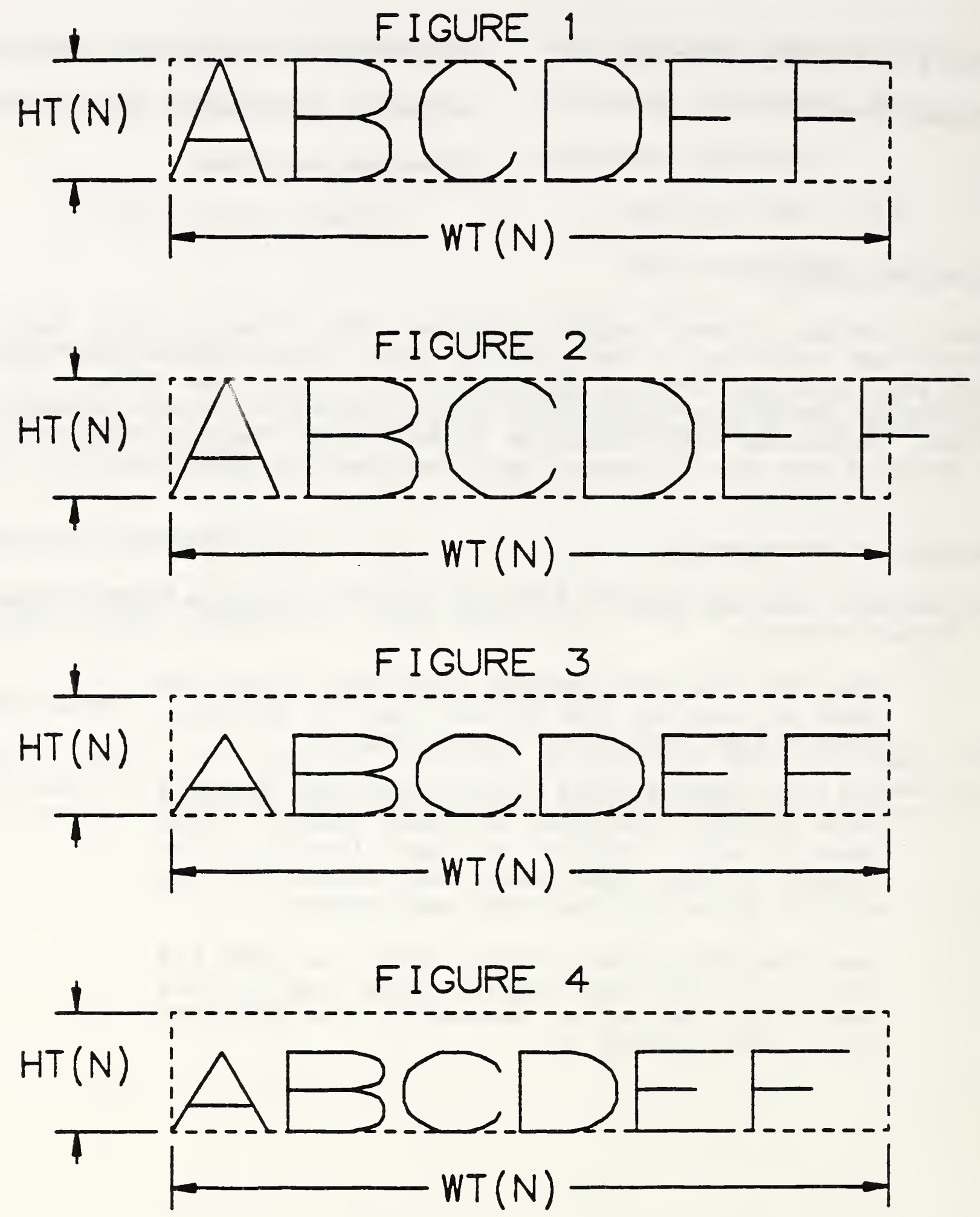


\section{Selected Methodology:}

Recommended Practices usually suggest only one way to process a particular portion of an entity. However, it was brought to the attention of the Recommended Practices Committee that different applications require different results. Implementors should study the applications being performed on their systems and should apply the appropriate method from above.

a. If more than one application is running on a particular system, an option should be made available which allows the user to choose the desired method.

b. If a switch cannot be provided, the order using the above methods should be:

Method 1. Define text within the box

$$
\text { (Figure 4) }
$$

Method 2. Define text within the width of the box (Figure 3)

Method 3. Define text within the height of the box (Figure 2) 
Category: Software Practice

Reywords: Text

General Note

Null string
IGES Document Version: All

Affected Processor: Both

Affected Entities:

General Note (212)

\section{Problem statement:}

Prior to IGES 5.0, the null string was not defined. The number of characters in a Hollerith string must be greater than zero; therefore, "OH," is an invalid Hollerith string parameter.

\section{Alternatives Considered:}

1. Define the null string as one blank character (i.e., "1H,").

2. Define the null string as a default parameter (i.e., ",,"). Most postprocessors cannot recognize a defaulted string parameter value.

\section{Selected Methodology:}

Preprocessors should write the null string as ",,".

Postprocessors should interpret a General Note Entity (212) with one string, where the string is either one blank text string ("1H ,") or a default parameter (","), as a null string. 
Category: Software Practice

Reywords: Text

General Note

Drafting Symbols
IGES Document Version: All

Affected Processor: Pre

Affected Entities:

General Note (212)

General Symbol (228)

\section{Problem statement:}

ANSI Y14.5 drafting symbols have fixed aspect ratios, and their widths do not correspond to normal ASCII alphanumeric characters. In the exchange of the General Symbol Entity (228), there are alignment problems when the symbols are used in the same strings with alphanumerics.

\section{Selected Methodology:}

Font Characteristics (FC) 1001, 1002, and 1003 contain both ANSI and non-ANSI special symbols. (Examples of non-ANSI symbols are " \pm ", "०", and Greek letters such as "pi".)

When ANSI non-alphanumeric symbols are used in General Notes (212), they should be used in strings of a single character, with a width as specified in ANSI Y14.5M-1982, Dimensioning and Tolerancing. 


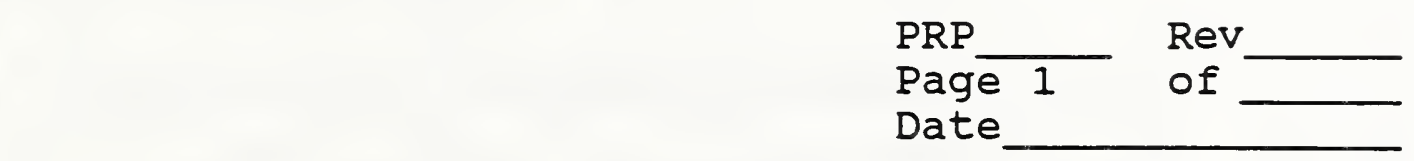

INITIAL GRAPHICS EXCHANGE SPECIFICATION

PROPOSED RECOMMENDED PRACTICE

Author:

Address :

Phone:

Ext.

Category:

Keywords :

Affected Processors: Pre/Post Affected Entities:

Topic:

Problem statement: 
PRP

Page

INITIAL GRAPHICS EXCHANGE SPECIFICATION PROPOSED RECOMMENDED PRACTICE (cont.)
Rev

of 
The following Edit Change orders (ECOS) to IGES Version 4.0 were approved by the IGES RFC Review committee and were incorporated into IGES Version 5.0 when it was published in october, 1990. They are summarized here for the benefit of those who may not have been aware of them. For a copy of the complete ECO, contact the IPO Chairman, National Institute of Standards and Technology, Bldg. 220, Rm. A127, Gaithersburg, MD, 20899.

E500 (RFC 361A)

E501 (RFC 375)

E502 (RFC 387B)

E503 (RFC 394)

E504 (RFC 358)

E505 (RFC 373A)

E506 (RFC 380)

E507 (RFC 283C)

E508 (RFC 340A)
FILE BLANK LINES - Specifies that IGES files may have trailing blank lines (e.g., to pad a disk block), but no leading blank lines.

PIECEWISE-COPIOUS PARAMETRIZATION - PrOvides parametrizations for the Copious Data Entity (Type 106, Forms $11 \& 12$ ).

NULL POINTERS - Clarifies situations in which defaulted or "null" pointers can be used.

PROCESS PLANT ATTRIBUTES - Adds predefined values to the Attribute Table Definition Entity (Type 322).

LINE FONT 0 - Adds a "no line font specified" value, analogous to the "no color specified" value.

CSG DISJOINT COMPONENTS - Creates a selected Component Entity (Type 182) for use in CSG applications.

EXTENDED VIEWS VISIBLE - Creates a new Form of the Associativity Instance (Type 402, Form 19) to indicate view visibility, color, and line-style of curves based on parameterization.

PERSPECTIVE VIEW - Creates a new Form of the View Entity (Type 410, Form 1) to transfer perspective views in a form similar to the presentation methodology of PHIGS.

REDUNDANT EXTERNAL REFERENCE - Deprecates the External Logical Reference File Index Associativity Entity (Type 402 , Form 2). 
E509 (RFC 369A)

E510 (RFC 374)

E511 (RFC 379D)

E512 (RFC 381/382)

E513 (RFC 383)

E514 (RFC 384)

E515 (RFC 388)

E516 (RFC 392)

E517 (RFC 398)

E518 (RFC 399)

E519 (RFC 404)

E520 (RFC 413)
PATTERN HATCH ENTITY - Defines new area fill pattern codes for the Sectioned Area Entity (Type 230) to support AEC applications.

COMPOSITE COPIOUS REFERENCE - The composite Curve Entity (Type 102) may now reference a Copious Data Entity (Type 106).

BOUNDED SURFACE - creates a new Bounded Surface Entity (Type 143) and a new Boundary Entity (Type 141).

LINEAR DIMENSION EXTENSIONS - AddS new Forms to the Linear Dimension Entity (Type 216) to indicate use as a Diameter Dimension (Form 1) or a Radius Dimension (Form 2).

GENERAL SYMBOL IEADER - Clarifies the use of the Witness Line Entity (Type 106, Form 40) and Leader/Arrow Entity (Type 214) in connection with the General symbol Entity (Type 228, Forms 1 \& 3).

GENERAL SYMBOL USER FORM - Reserves implementor-defined Form Numbers for the General Symbol Entity (Type 228).

FEM ELEMENT ADDITION - Five new topology types are added to the Finite Element Entity (Type 136).

PLANE/SINGLE PARENT - Deprecates the use of the single Parent Associativity (Type 402, Form 9) to create holes in bounded planar regions.

COMPRESSED ASCII REVISION - ReVised definition of the compressed ASCII Format for IGES files.

TERMINATE REVISION - Revised definition of the Terminate section.

MATRIX ORDER - corrects explanation of inténded operation of explicitly nested Transformation Matrix Entities (Type 124).

POINT DIMENSION EXTENSION - Allows the Point Dimension Entity (Type 220) to reference a Simple closed Planar Curve Entity (Type 106, Form 63). 
E521 (RFC 418)

E522 (RFC 419A)

E523 (RFC 425)

E524 (RFC 428)

E525 (RFC 429)

E526 (RFC 430)

E527 (RFC 389)

E528 (RFC 390)

E529 (RFC 335)

E530 (RFC 370C)

E531 (RFC 414A)

E532 (RFC 432A)

E533 (RFC 436)
VIEWS VISIBLE ENTITY COUNTS - The pointers to entities which are affected by the Views Visible Associativity Entities (Type 402, Forms 3 \& 4 ) are no longer required; for backward compatibility, they are optional.

B-SPLINE WEIGHTS - Clarifies the definition of a rational B-spline.

SPICE PARAMETERS - Adds predefined attributes for electrical applications to the Attribute Table Definition Entity (Type 322).

COMPOSITE CURVE RESTRICTIONS - clarifies a special case of the Composite Curve Entity (Type 102) in regards to the connect point Entity (Type 132).

SIMPLE CLOSED CURVE - Adds a definition of a simple closed Curve to the Glossary.

106/63 CLARIFICATION - The simple Closed Area Entity (Type 106, Form 63) has been renamed Simple Closed Planar Curve.

TABULAR DATA PTYPE=12 - Clarifies Nodal Loads/Constraint Data in the Tabular Data Form of the Property Entity (Type 406, Form 11).

UNITS DATA ENTITY - Creates a Units Data Entity (Type 316) for use in FEM applications.

BINARY TO APPENDIX - Deprecates the Binary Format for IGES files.

PREDEFINED LINE FONT PATTERNS - Creates a new Form of the Property Entity (Type 406, Form 19) to identify AEC line font patterns.

8-BIT ASCII - Clarifies the prohibition against 8-bit ASCII characters in IGES files.

USER/IMPLEMENTOR DEFINED - Changes all references of "user defined" to "implementor defined".

VIEW CLIPPING PLANES - Permits use of the Unbounded Plane Entity (Type 108, Form 0) for clipping planes referenced by a View Entity (Type 410). 
E534 (RFC 377B)

E535 (RFC 316)

E536 (RFC 372)

E537 (RFC 376)

E538 (RFC 378B)

E539 (RFC 406)

E540 (RFC 431)

E541 (RFC 440)

E542 (RFC 442)

E543 (RFC 445A)

E544 (RFC 446)

E545 (RFC 448)
HIGHLIGHT AND PICK PROPERTIES - Creates two new Forms of the Property Entity (Type 406, Forms $20 \& 21$ ) to communicate "highlight" and "pick".

DIMENSION ORIGIN LEADER (214/12) - Creates a new arrowhead style for the Leader (Arrow) Entity (Type 214).

B-REP SURFACES - Creates new entities for Boundary Representation (B-REP) surfaces.

(This ECO was canceled by E587.)

UNIFORM RECTANGULAR GRID PROPERTY (406/22) Creates a new Form of the Property Entity (Type 406) to exchange rectangular grids on drawing sheets.

DRAWING WITH ROTATED VIEWS (404/1) - Creates a new Form of the Drawing Entity (Type 404) to exchange drawings with rotated views.

APPENDIX SAMPLE FILE - Replaces the Electrical Part Sample file in Appendix $A$ of the specification with the file from the Electrical Application Guide.

NULL STRING - Adds an entry to the Glossary Appendix to define the Null string.

NEW ORDINATE DIM FORM \# $(218 / 1)$ - Creates a new Form for the ordinate Dimension (Type 218) containing both a Witness Line and a Leader (Arrow).

CONNECT POINTS IN NETWORK SUBFIGURES Creates an explicit linkage between the connect point entities (Type 132) in a Network Subfigure Definition entity (Type $320)$ and the Network subfigure Instance entities (Type 420) which reference it.

STANDARD BLOCK FONT - Changes the name of font characteristic (FC) 1 in the General Note Entity (Type 212).

GLOBAL PARAMETER 16 DEFAULT - Provides a default value for Global parameter 16 (Maximum Number of Line Weight Gradations).

RENAME GLOBAL PARAMETER 17 - Changes the name of Global Parameter 17 from "Size of maximum 
E546 (RFC 395A)

E547 (RFC 437E)

E548 (RFC 451)

E549 (RFC 454)

E550 (N/A)

E551 (N/A)

E552(N/A)

E553 (N/A)

E554 (N/A) line width" to "Width of maximum line weight".

CONNECTIVITY DEFAULT POINTERS - Clarifies the descriptions of certain pointers in Entity Types 132,320 , and 420 .

KANJI GENERAL NOTE - Adds support for the Japanese national character set (JIS C 62261983) as a new font characteristic (FC 2001) in the General Note Entity (Type 212).

MACRO ENTITY TO APPENDIX - MOVe the definition of the Macro Entity (Type 306) to the Unimplemented Entities ("Gray Page") Appendix.

PLACEMENT OF DRAFTING SYMBOLS - Adds a figure giving examples of text-height and text-width as it applies to the display of drafting symbols defined in ANSI Y14.5M-1982 Dimensioning and Tolerancing.

ERRATA FROM VERSION 4.0 - (editorial tracking) The IGES Editor is directed to add the errata for IGES Version 4.0 to the Working Draft for the next version.

REORGANIZE AND ORDER ENTITIES - (editorial tracking) The IGES Editor is directed to reorganize the working Draft of the next version as needed to accomplish the goal of listing the entity definitions in ascending order by Entity Type Number.

CONSISTENCY OF PD SUBSECTIONS - (editorial tracking) The IGES Editor is directed to make consistent appearance of vertical dots, the phrase "or zero", and the phrase "Pointer to the DE of the ..." in the PD subsections of each entity definition.

CORRECTIONS TO EXAMPLE PART FILE - (editorial tracking) The IGES Editor is directed to add a corrected version of the Appendix A Example 3 IGES file (Drawing and View Example).

CHANGE BARS - (editorial tracking) The IGES Editor is directed to devise a practical method of indicating ECO changes since Version 4.0 of the specification. 
REMOVE SUBSECTION NUMBERS ON DE \& PD (editorial tracking) The IGES Editor is directed to remove the subsection numbers from $D E$ and $P D$ descriptions in the entity definitions.

E556 (N/A)

$E 557(\mathrm{~N} / \mathrm{A})$

E558 (N/A)

E559 (N/A)

E560 (N/A)

E561 (N/A)

E562 (N/A)

E563 (N/A)
CORRECT FIGURE 78 - (editorial tracking) Figure 78 is moved to the Grey Page Appendix and replaced by a correct figure.

PARAMETRIC SPLINE CURVE - (editorial tracking) An error occurred in transcribing Version 3 to Version 4; in Section 3.8 (page 91), in the sentence that begins "In order to avoid degeneracy, ...", the phrase, "... must be zero:" should be, "... must be nonzero:".

AMEND ECO 506 (EXTENDED VIEWS VISIBLE) (editorial tracking) In section 4.3.11 (page 339), in the last paragraph, change the phrase, "(Type 402, Form 3 or 4)" to "(Type 402 , Form 3, 4, or 19)".

PARAMETER DATA LISTS - (editorial tracking) Corrects the Parameter Data Lists of a few entities for editorial consistency.

AMEND ECO 511 (BOUNDED SURFACE) - (editorial tracking) Several of the parameter names for Entity Types 141 and 143 were confusing.

MOVE TYPES $322 \& 422$ FROM GRAY PAGES TO BODY - (editorial tracking) The material for Entity Types 322 (Attribute Table Definition) and 422 (Attribute Table Instance) is moved from Appendices J.8 and J.9 into the appropriate locations in the body of the specification.

MOVE FC 1003 FROM GRAY PAGES TO BODY (editorial tracking) The material for Font 1003 (Drafting Font) is moved from Appendix J.5 into section 4.2.9 General Note Entity (Type 212).

APPENDIX SAMPLE FILE (A.2) - (editorial tracking) In the Mechanical part Example (Appendix A, Example 2) the setting of the Hierarchy Flag on some of the dimensions caused several postprocessors to produce incorrect results. 
E564 (RFC 189B)

E565 (RFC 256B)

E566 (RFC 386C)

E567 (RFC 405E)

E568 (RFC 407B)

E569 (RFC 416B)

E570 (RFC 447A)

E571 (RFC 450A)

E572 (RFC 452)

E573 (RFC 455A)

E574 (RFC 459A)
POINT PARAMETER 4 - In the Point Entity (Type 116), parameter index 4 (PTR) is changed from "subfigure instance" to "subfigure definition."

MODEL DATE/TIME STAMP - A new parameter is added to the Global section to record the date/time of the native model's creation or last modification.

CURVE DIMENSION ENTITY (204) - Creates a new Curve Dimension Entity (Type 204) for use in drafting applications.

IMPROVED GENERAL NOTE (213) - Creates a New General Note Entity (Type 213) for the representation of text strings, as an alternative to the Type 212 .

CONFORMANCE RULES - A definition of conformance is added to the specification.

SECTIONED AREA ENTITY (230/0) - Clarifies the definition of the Sectioned Area Entity (Type 230, Form 0).

NEW 230 FORM NUMBER (230/1) - Creates a new Form Number of the sectioned Area Entity (Type 230/1) for Inverted Crosshatching.

ASSOCIATIVITY GROUP TYPE PROPERTY (406/23) Creates a new Form Number of the Property Entity (Type 406, Form 23) for naming Group Associativities.

NEW EXTERNAL REFERENCE FORM NUMBER (416/3) Creates a new Form Number of the External Reference Entity (Type 416, Form 3) for referencing native parts instead of IGES files.

LEVEL TO PWB LAYER MAP (406/24) - Creates a new Form Number of the property Entity (Type 406, Form 24) for mapping exchange file levels to printed wire Board layers.

PWB ARTWORK STACKUP (406/25) - Creates a new Form Number of the Property Entity (Type 406 , Form 25) for representing Printed wire Board Artwork. 
E575 (RFC 461A)

E576 (RFC 462A)

E577 (RFC 464A)

E578 (RFC 465A)

E579 (RFC 469A)

E580 (RFC 473A)
E581 (RFC 477A)

E582 (RFC 479A)

E583 (N/A)

E584 (N/A)

E585 (N/A)
PWB DRILLED HOLE $(406 / 26)$ - Creates a new Form Number of the Property Entity (Type 406, Form 26) for representing drilled holes in Printed Wire Boards.

CONNECT POINT EXTENSION - Adds new Pin Type and Pin Function values to the connect point Entity (Type 132).

REGION RESTRICTION EXTENSION - AddS new constraints to the Region Restriction property (Type 406, Form 2).

PWA MANUFACTURING ATTRIBUTES - Adds a new Attribute List Type $(A L T=5)$ to the Attribute Table Definition Entity (Type 322) for Electrical and Printed Wire Assembly Manufacturing.

GENERAL NOTE TEXT BOX - Removes the requirement that text box height be preserved by postprocessors.

DE REQUIREMENTS - Removes Table 3 and places the DE requirements for each entity along with its PD description.

CHANGE ENTITY TYPE NUMBERS - Changes several of the Entity Type Numbers assigned by previous Edit Change orders.

B-SPIINE CLOSURE PROPERTY - Clarifies the term "closed" for Rational B-spline Curves (Type 126) and Rational B-spline surfaces (Type 128).

MOVE NULI ENTITY FROM GRAY PAGES - (editorial tracking) The material for the Null Entity (Type 0) is moved from Appendix J.2 into the body of the specification.

MOVE 214/12 ENTITY FROM GRAY PAGES (editorial tracking) The material for the Dimension origin arrowhead style (Type 214, Form 12) is moved from Appendix $J$ into the body of the specification.

DELETE APPENDICES C \& D (editorial tracking) The material in Appendices C (Plant Flowsheet Representation) and D (Piping Model Example) is removed from the Specification. 
E586 (N/A)

E587 (N/A)

E588 (N/A)

E589 (N/A)

E590 (RFC 392)

E591(N/A)

E592 (RFC 473)
DELETE APPENDIX B - (editorial tracking) The material in Appendix B (Electrical/Electronic Product Representation) is removed from the specification.

DEPRECATE ECO 536 (B-REP SURFACES) (editorial tracking) ECO 536 is cancelled; the material must be reballoted as an RFC.

CONFORMANCE RULES CORRECTION - ECO 568 contained the incorrect text; it was the text as balloted, not as amended by the ballot review. This is the correct text, and this ECO supersedes ECO 568.

PD LISTS FOR COPIOUS DATA (TYPE 106) Several ECOs make the current format of the various forms of the Copious Data Entity (Type 106) impractical to print as in previous versions of the specification.

AMEND ECO 516 (PLANE/SINGLE PARENT) - ECO 516 did not provide sufficient editorial instructions to preserve the upward compatibility of the specification.

CLARIFICATION OF BOUNDED PLANES - In the description of the Plane Entity (Type 108), the text is not consistent with the restrictions on the Form Numbers and PD field 5 .

AMEND ECO 580 (DE REQUIREMENTS) - DE requirements for two of the Entity Types (134 and 422 ) contained errors that contradicted the text. 


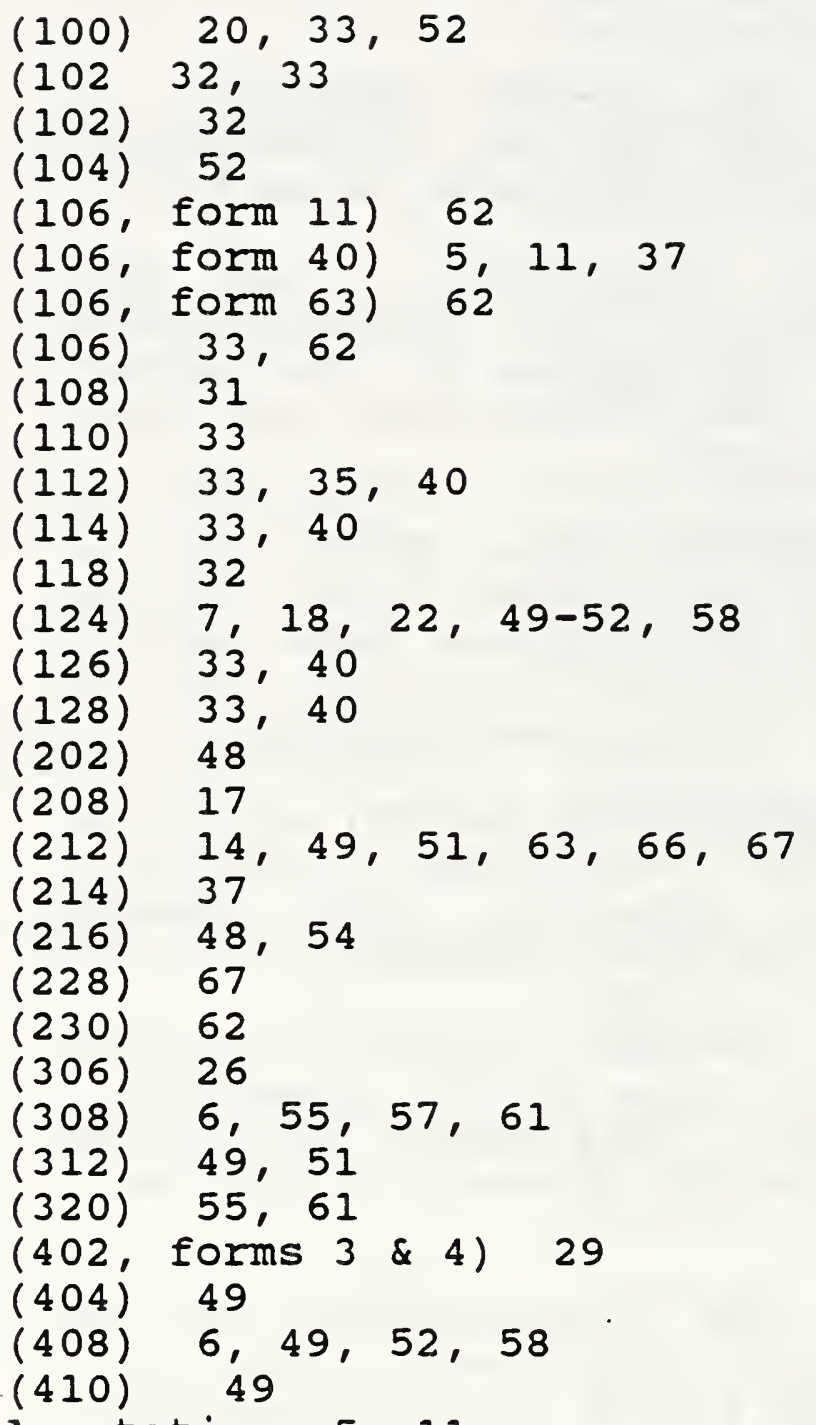

Annotation 5, 11

Arrowhead $37,48,74,79$

Blank Status 5,47

Comment 30

Coordinate System 18

DE Hierarchy Flag 47

$D E$ Level Number 11,38

Default value $42-46,75$

Directory Entry 5-7, 27, 30, 47, 54

Documentation 2, 12, 15, 19, 37, 46

Drafting Symbols 67

Drawing 47, 49, 74, 76

Entity Mapping $2,11,31,33,37,38,40$

Entity Origin 49,52

Error Message 12, 13, 19

Extra View 18

Flag Note 17

Global Parameter $8,10,24,25,35,42,43,53,75$ 
Global section $8,10,24,35,42,77$

Hierarchy $2,6,27,47,77$

Hierarchy Flag 47,77

Hierarchy Rule 2, 6, 27, 47

Identity Matrix 7, 49

IGES Description 2, 8, 17, 26, 29

Implementation Rationale 2

Index 81

Information Message 15, 19

Invalid Data 20

Leader Line 28,37

Level Number $11,38,47$

MACRO 26, 75

Manual 1, 2, 19

Matrix 6, 7, 18, 20, 22, 23, 27, 49-52, 58, 72

Matrix Processing 6, 7, 18, 22

Max. Coordinate Value 10, 43

Minimum Resolution 35

Model Space Scale 24, 43, 44

Multiple Pass 39

Names 55, 59-61, 76

Null string 66,74

Number Format 53

origin 49,52

$\mathrm{PD} 26,30,54,55,59,60,75,76,78,80$

PDDI Report 18, 19, 24, 26, 37

Performance iii, 2, 7, 35

Plane $31,52,72,74,80$

Required Pointers 57

Robustness 12

Rotation Angles 51,52

Sectioned Area Entity 62, 72, 77

Single Pass 39

Slope Discontinuous 32

Software Practice $2,4,5,9,10,18,20,22,24,30,32$,

$$
39,42,48,49,51-55,57-59,61-63,66,67
$$

Spline Curve $33,35,40,41,76$

spline Surface 33,40

Subfigure $6,55,57$

Subord. Entity Switch 11, 27

Summary Report 15

System ID 8,43

Tape Format 9

Testing Method 2, 36

Text $2,14,17,25,49,51,53,63,65-67,75,77,78,80$

Thrashing 54

Transformation Matrix 7, 18, 20, 22, 49-52, 58, 72

View $18,29,47,49,58,71,74,76$

View Associativity 29

Witness Line 5, 11, 28, 37, 48, 72, 74

Workaround 2 

NIST.114A

(REV. 3-0)
U.S. DEPARTMENT OF COMMEACE

NATIONAL INSTITUTE OF STANDARDS AND TECHNOLOGY

BIBLIOGRAPHIC DATA SHEET
1. PUCUCATION OR REPORT MUMLER NISTIR 4600

2 PERORMIMO ORCWMIZTION REPORT MUMBEA

3. RURUCATION DAT

\section{TISANO SURTा}

IGES 5.0 Recommended Practices Guide

S WTHOn(3) IGES Recommended Practices Committee, Linda Martino, Chairman; Dennette A. Harrod, Jr. IGES Project Manager; William Conroy, Chairman, IGES/PDES Organization; Joan Wellington, Editor IGES/PDES Organization

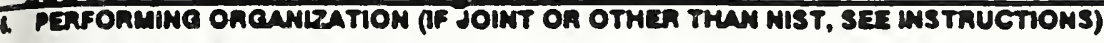

U.S. Desantruart of COMMERC:

MATIONA INSTIUUTE OF STANDANOS ANO TECANOLOOY

cNTHEAswina, no 2000

\section{CONTRACT/ORATT MUMEER}

Q TMPE OF REPOAT AND PERIOD COVEAED Final; $12 / 88-3 / 91$

1. SPONSORIMG ORCWIRATION MAME AND COMPLTTE ADDRESS (STAET, CTY, STATE, 2A)

Factory Automation Systems Division

NIST

Building 220, Room A-127

Gaithersburg, Maryland 20899

10. SUPFLEIENTARY MOTES

N/A

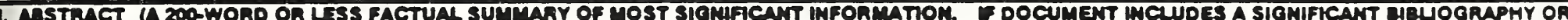
UTIZATURE SUAVEY, METION $T$ HISRE)

The IGES 5.0 Recommended Practices Guide has been written to assist both users and implementors of the Initial Graphics Exchange Specification (IGES). It contains two types of information: explanations of implementation practices that are alternatives to those documented in the Specification and clarifications of ambiguities or omissions in the Specification.

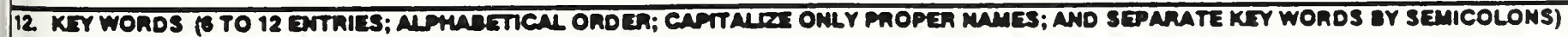

Initial Graphics Exhchange Specification (IGES), Recommended Practices, affected entities, affected processors, alternatives, selected methodology.

\section{AVALAIITY}

unaurme

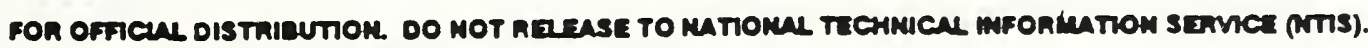

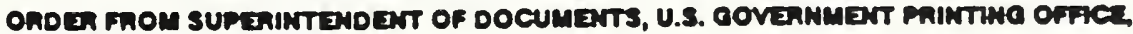
WASHUMOTON, DC 20102.

ORDEA FHOM MATIONLL TECHMICN IMFORMATION SEMVCE (MTS), SPAINOFED, VA 20161.
14. MUMLEA OF PRIKTED PAOES

91

\section{ELECTRONIC FORM}




